\title{
Determining the Young's modulus and creep effects in three different photo definable epoxies for MEMS applications
}

\author{
Kristof Wouters*, Robert Puers \\ K.U.Leuven, Department Elektrotechniek ESAT-MICAS, Kasteelpark Arenberg 10, B-3001 Leuven, Belgium
}

\section{A R T I C L E I N F O}

\section{Article history:}

Received 30 September 2008

Received in revised form 31 March 2009

Accepted 31 March 2009

Available online $\mathrm{xxx}$

\section{Keywords:}

Young's modulus

Creep

Epoxy

Epoclad

Epocore

\begin{abstract}
A B S T R A C T
In this study, a fundamental measurement of the Young's modulus of Epoclad and Epocore negative photo resist is performed, using a miniature tensile test setup. The experiments were done on miniature dog bone tensile test structures which are fabricated using normal micro machining methods. In addition, the creep behavior and the maximum stress and strain at the point of fracture of the resists is recorded. The two resists reveal a mechanical behavior very similar to the well-reported resist SU-8. SU-8 is also tested using the same tools for comparison.
\end{abstract}

(c) 2009 Elsevier B.V. All rights reserved.

\section{Introduction}

Recently, part of the research in MEMS technology is being reoriented towards other materials, besides silicon or other semiconductor compounds. Such new materials give the possibility to further improve MEM sensors and actuators and can also open up an entirely new application domain for MEMS. Polymer based compounds are a group of materials that are very promising. Using their broad spectrum of chemical and mechanical properties they can enable new and cheaper MEMS [1-3].

From the mechanical point of view, epoxy based materials are good candidates to be used as structural layers in a MEM structure. Epoxies are typically relative stable, both chemically and mechanically. They are classified as thermo sets. Because of the crosslinks in the polymer network, the epoxies under investigation will burn at high temperatures before any significant melting occurs. Epoclad and Epocore are, as SU-8, epoxy based resist which promises good mechanical properties. These commercially available compounds are UV curable and thus easy to process using standard lithography equipment.

Epoclad and Epocore find there origin in the fabrication and integration of optical wave guides [4]. In this way, optical interconnects can be integrated at the printed circuit board level. It enables the possibility to combine high bandwidth communication lines together with the conventional copper interconnects. The

\footnotetext{
* Corresponding author. Tel.: +32 16321716; fax: +32 16321975

E-mail address: Kristof.wouters@esat.kuleuven.be (K. Wouters).
}

Epocore resist is optimized for the core of a wave guide, while the Epoclad is optimized for the cladding, whence the name. Fig. 1 displays an in situ fabricated optical splitter using these materials [5].

It is clear that the mechanical properties for Epoclad and Epocore are rather irrelevant in such optical applications. However, if one wishes to use these materials for mechanical applications, as a structural component, the knowledge of theses mechanical properties become imperative. To the authors' knowledge, no mechanical properties have been reported yet on these resists.

The Young's modulus is measured using a miniature tensile test tool. The setup is able to measure force when a given displacement is applied on miniature samples. In this way, the Young's modulus can be determined in a straight forward and fundamental manner. The creep of the epoxy material is also tested. The definition of creep in this paper is as follows: creep is the deformation (not necessarily permanent) occurring with a delay with respect to the moment of applied engineering strain or stress. In contrast, in metallurgy creep usually refers to a plastic, permanent deformation. Fig. 2 illustrates this definition with an example where an arbitrary visco-elastic sample is loaded with a certain engineering strain. At time zero a constant engineering strain is applied. A stress will result, that however decreases in time due to the visco-elastic properties of the material. The material will creep and relax some of its stress. When, after some time, the engineering strain is set to zero again, a compressive force is needed to counteract the deformation during the creep phase. This force will drop to zero in time. The material recuperates or restores its original state. 


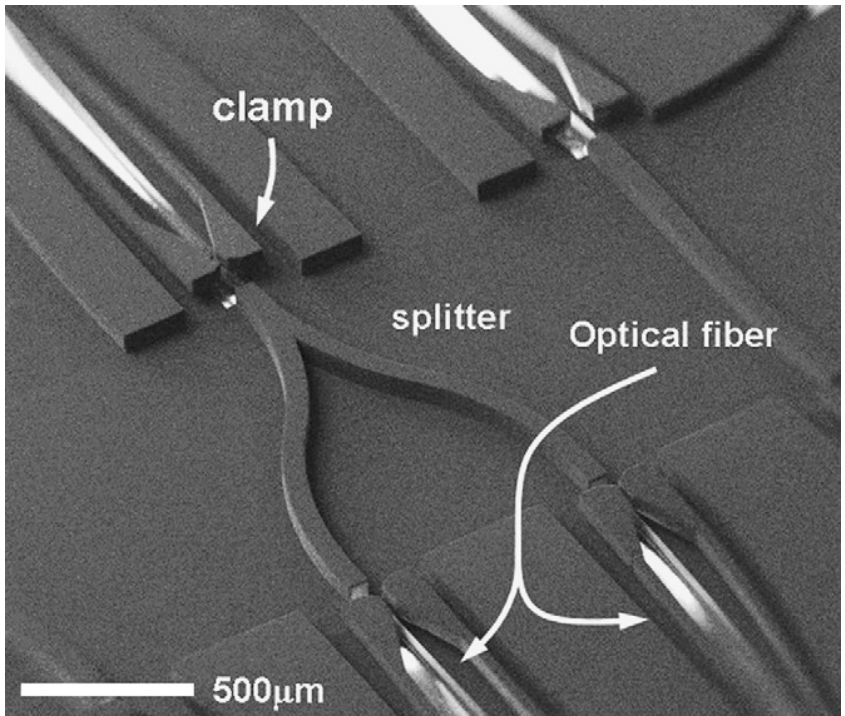

Fig. 1. Optical splitter fabricated using Epoclad as the cladding and Epocore as the core. The top cladding, or Epoclad layer is left off for the clarity of the picture. Also a clamping structure is processed in the same material as the core material for clamping and aligning the glass optical fibers.

\section{Experimental details}

\subsection{Tensile test setup}

The setup used is a delaminator test system from the DTS Company, (Menlo Park, CA), fitted with custom made sample mounting clips to mount the test samples. The force is measured using a load cell featuring a non-linearity of $0.05 \%$, a hysteresis of $0.03 \%$ and a creep of $0.025 \%$ in a $20 \mathrm{~min}$ time frame. The actuator used to apply the force and to measure the displacement features a minimum increment motion of $100 \mathrm{~nm}$ and a bidirectional repeatability of $1 \mu \mathrm{m}$. Fig. 3 shows the miniature tensile tester. Control and data acquisition is done using a PC.

\subsection{Fabrication}

Three different polymers will be investigated in this study: SU-8, which is best characterized to this moment, Epoclad and Epocore.

SU-8 2050 is bought from Microchem. Corp. Epoclad and Epocore were bought from Micro Resist Technology GmbH. For fabricating the samples in Epoclad and Epocore LOR 30B (lift off resist) from Microchem. Corp. was used as a sacrificial layer. For the SU-8 samples Teflon foil was used as a substrate. The FEP (fluorinated

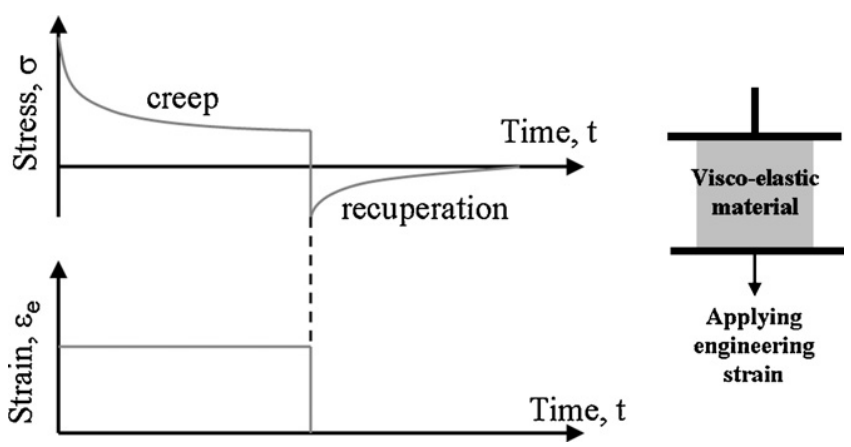

Fig. 2. An engineering strain on a cubic shaped piece of visco-elastic material will result in a instantaneous stress. This stress will drop over time and is defined as creep. When the cube is pushed back to its original dimension, a compressive force is required, that will drop to zero over time. This is defined as recuperation.

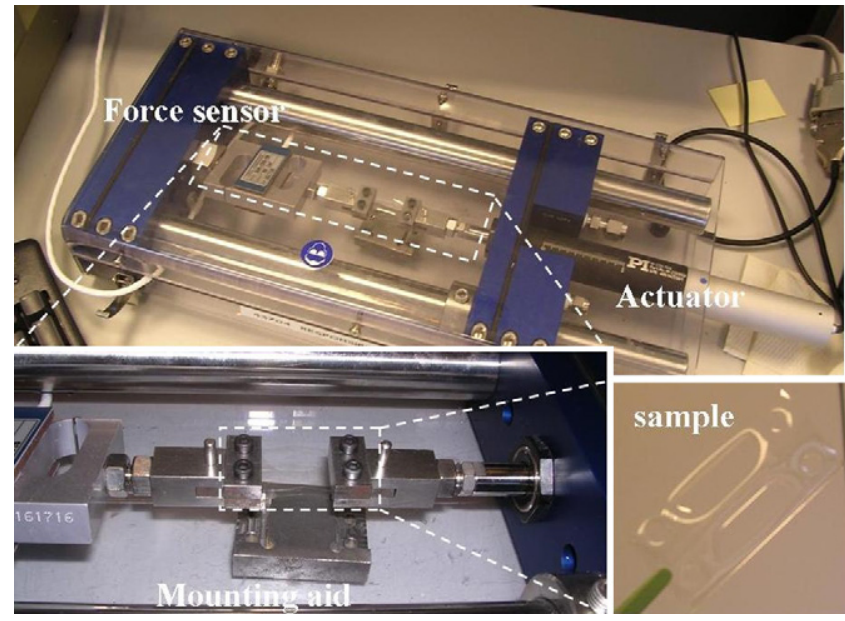

Fig. 3. Delaminator fitted with sample mounting clip for the tensile tests.

ethylene propylene) Teflon foil 500A (127 $\mu \mathrm{m}$ thick) was obtained from Katco Ltd.

The SU-8 2050 is spin coated directly on to a piece of the FEP Teflon foil at $3000 \mathrm{rpm}$ and soft baked on a leveled hotplate at $70^{\circ} \mathrm{C}$ for $90 \mathrm{~min}$. After a cool period of approximately $30 \mathrm{~min}$ the sample is exposed with $320 \mathrm{~mJ} / \mathrm{cm}^{2}$ of UV light (measured at $400 \mathrm{~nm}$ wavelength). Wavelengths below $360 \mathrm{~nm}$ are filtered out. A post bake is done in a conventional oven under a nitrogen atmosphere at $80^{\circ} \mathrm{C}$ for $40 \mathrm{~min}$. Next, the Teflon SU-8 stack is immerged in PGMEA (propylene glycol monomethyl ether acetate) to dissolve the non-cured SU-8 and rinsed in isopropyl alcohol. The SU-8 dog bone structure can now be peeled from the Teflon foil. However, to avoid damage to the structure, a 10 min ultrasonic bath immersion in water is used to separate the SU-8 from the Teflon foil. A final hard bake is performed by placing the free standing dog bone structures in a conventional oven under a nitrogen atmosphere for typically $60 \mathrm{~min}$ at $150^{\circ} \mathrm{C}$ temperature, see Fig. $4 \mathrm{~A}$.

For the realization of the Epoclad and Epocore dog bone structures the Teflon foil can not be used with these resists, because of the reflow occurring during the soft bake step. Instead, a 3-in. silicon wafer coated with LOR $30 \mathrm{~B}$ is used as carrier substrate. The LOR $30 \mathrm{~B}$ is spun at $2000 \mathrm{rpm}$ and cured on a leveled hotplate for $5 \mathrm{~min}$ at $170^{\circ} \mathrm{C}$. Subsequently, Epoclad or Epocore is spun at $2000 \mathrm{rpm}$ and baked according to the datasheet provided by the manufacturer, except the soft bake time was increased to avoid stiction
(B)

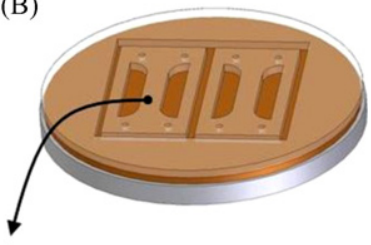

(C)

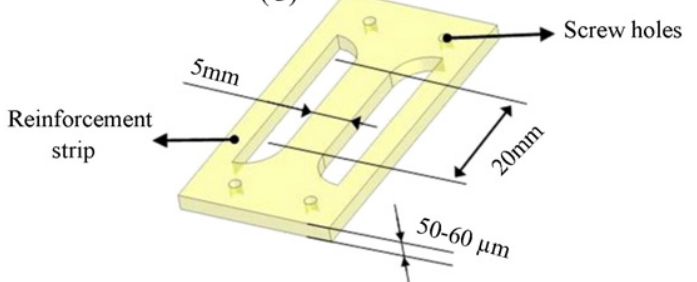

Fig. 4. Schematic of the fabrication process. (A) SU-8 fabrication process using Teflon foil as a substrate, (B) the Epoclad/Epocore fabrication using Si substrate and a polyimide sacrificial layer. (C) The dog bone structure of the sample. The reinforcement strips were cut prior to measurement. 


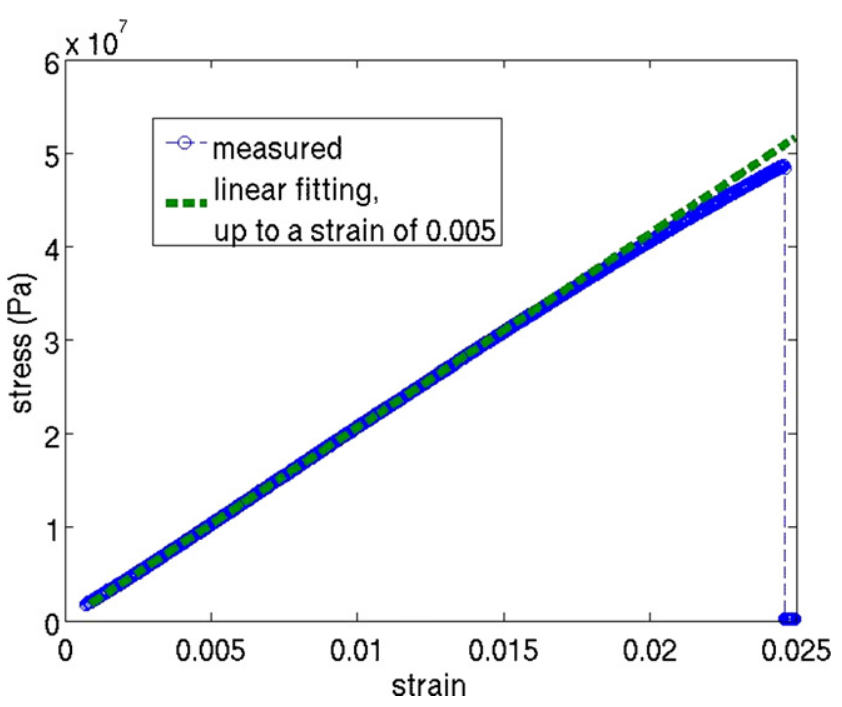

Fig. 5. Measured stress-strain curve of an Epoclad sample. It displays a typical epoxy-like behavior with brittle fracture. The strain calculated is the engineering strain.

to the mask. After soft bake, the film is exposed with $320 \mathrm{~mJ} / \mathrm{cm}^{2}$ UV light and cured inside a conventional oven under a nitrogen atmosphere for $60 \mathrm{~min}$ at $110^{\circ} \mathrm{C}$. Development was achieved using PGMEA following by rinsing in isopropyl alcohol. The dog bone structures were released from the silicon wafer by etching the LOR layer using a alkaline solution. As a last step the samples were hard baked for $60 \mathrm{~min}$ in an oven at $140^{\circ} \mathrm{C}$ under nitrogen atmosphere, see Fig. 4B.

\section{Experimental details and results}

\subsection{Young's modulus and maximum macroscopic stress and strain}

The measurements were carried out by first applying a considerable load to take up slack (e.g. from the fixation points), followed by reducing again the load to zero before the actual measurement. Because the epoxies are visco-elastic the measured Young's modulus is dependent on the speed of applied strain. The material will appear stiffer when stretched faster. So, the speed is kept constant for all measurements at $3 \mu \mathrm{m} / \mathrm{s}$, which is considered to be relative slow for samples that are $20 \mathrm{~mm}$ long.

Fig. 5 shows a measured stress-strain curve. A typical epoxy-like behavior with bridal fracture is observed for al three photo epoxies measured [9]. In Table 1 a summary is given of the obtained Young's moduli and the stress and strain upon fracture. It illustrates, to the authors' knowledge, the first Young's moduli and strength data of Epoclad and Epocore. Our measured SU-8 Young's modulus agrees very well with the $2 \mathrm{GPa}$ given in the manufacturer's datasheet of the SU-8 2000 series. A large number of samples were measured to cope with the large spread of the stress and strain upon fracture. In the following text this stress and strain upon fracture will be referred to as the maximum stress and strain.

Table 1

Summary of measured Young's modulus, maximum stress and maximum strain. The error indicated is the standard deviation.

\begin{tabular}{llll}
\hline & $E(\mathrm{GPa})$ & $\sigma_{\max }(\mathrm{MPa})$ & $\varepsilon_{\max }(\%)$ \\
\hline SU-8 & $2.1 \pm 0.2$ & $22 \pm 7$ & $1.2 \pm 0.5$ \\
Epoclad & $2.2 \pm 0.1$ & $43 \pm 10$ & $2.3 \pm 1$ \\
Epocore & $2.4 \pm 0.2$ & $46 \pm 10$ & $1.6 \pm 0.3$ \\
\hline
\end{tabular}

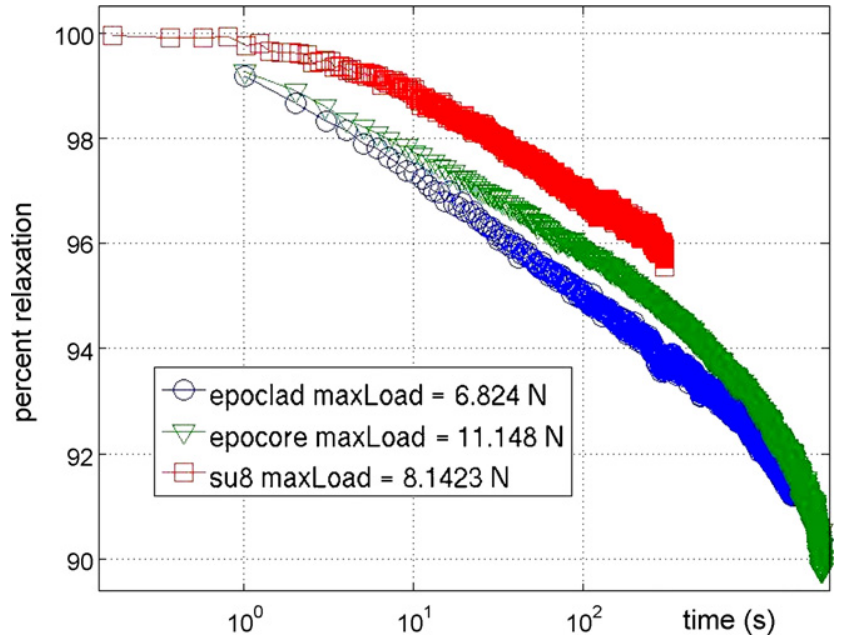

Fig. 6. Semi-logarithmic plot of the relaxation behavior of the three photo epoxies A constant strain is applied and the obtained load is given in the legend box. The load drops in time.

\subsection{Creep behavior experiment}

With the same setup, the relaxation in time, when a constant engineering strain is applied, can be measured. In Fig. 6, a semi-logarithmic plot of the relative relaxation with respect to the maximum load is depicted. The strain was applied, after tacking up slack, from zero to its maximum at a speed of approximately $200 \mu \mathrm{m} / \mathrm{s}$. The relaxation displays a fairly exponential behavior, as could be expected. The creep is plotted as a percentage of the force drop over the measured time, so the percentage creep is given by:

percent creep $=\frac{\operatorname{load}(t)}{\max \text { load }} \times 100$

With the strain removed, the samples shows some recuperation from the creep step, as shown in Fig. 8. The samples measured are thin films. This means that the sample cannot take compressive stress. It will buckle when the deformation is negative. To be able to measure the recuperation, some small residual tensile stress must be left on the thin film sample, to avoid that the sample buckles. The recuperation in Fig. 8a, is given in percent load increase with respect to the residual tensile load left on the sample.

percent recuperation $=\frac{\operatorname{load}(t)}{\text { residual load }} \times 100$

\section{Discussion}

\subsection{Young's modulus and maximum macroscopic stress and strain}

In Fig. 4C the dimensions of a tensile test sample are depicted. It is clear that the dimensions are orders of magnitude larger than those used in micro scaled devices or structures, except the thickness. Indeed, one has to consider the size dependency of the mechanical properties. When characterizing semiconductor based materials for MEMS the crystal structure and increased surfaces effects are the major cause of these size dependent properties [6,7]. An obvious example is the difference of the mechanical properties between bulk materials and thin film materials. The fabrication process also has an important influence on the mechanical properties of thin films. They will determine eventually the crystal structure (for instance amorphous or poly-crystalline) and the stresses in the film (for instance stresses caused by thermal mismatches).

For the epoxy based polymers under investigation, we assume that the spin coated thin films are amorphous and isotropic. Due to the typical high internal stresses in the epoxy films after curing, 


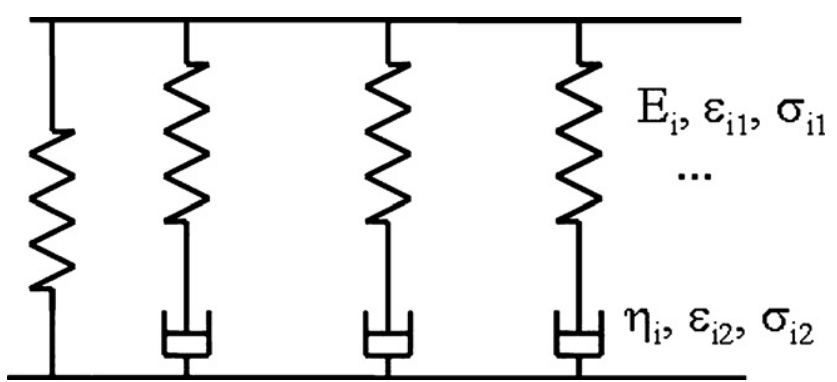

Fig. 7. Schematic representation of a Maxwell model of a linear visco-elastic solid. All elements are linear springs, with response: $\sigma=E \varepsilon$ or dashpots, with response: $\sigma=\eta \mathrm{d} \varepsilon / \mathrm{d} t$. The first branch ensures that the modeled material does not display any permanent deformation.

the assumption that they are isotropic may not be correct. Nevertheless, the tensile test samples allow the measurement of the in-plane Young's modulus which is the most important in view of the creation of MEM structures. The fabrication process of the test samples uses standard micro machining techniques. Also, the thickness of the sample is in the order of tens of micrometers. This is done in order to stay close to the fabrication process of polymer MEM structures. In this way, thickness and process dependencies of the mechanical properties get eliminated as much as possible. Depending on the curing time and temperature the properties of SU-8 can vary significantly. This is no more the case when the curing reaction saturates and the epoxy is fully cured [8]. The same behavior is also observed for Epoclad and Epocore but not yet studied in dept because we wish to focus on the fully cured and most stable condition of the polymer.

An important remark must be made on the maximum stress and maximum strain in Table 1. Failure is a defect driven phenomenon. Fracture will occur at a critical defect somewhere in the sample. For instance, due to foreign contaminants or cracks that are randomly distributed, with an average density that depends on the process conditions. Because these samples are macroscopic in size, they will fail at lower stresses than micro scale structures [10].

\subsection{Creep behavior}

In our experiments the creep behavior was recorded for, on average, half an hour. A further significant increase of the recording time would be impractical using this setup. From a MEMS point of view it is very important to know if the material deforms permanently. The occurrence of permanent deformation, for instance caused by thermal induced stresses in the structural layer, is a big reliability problem in future devices. In order to find out that the creep is a permanent deformation or that the material will snap back with time, a phenomenological model is used.

The creep behavior is used to setup a model of a material that does not have permanent deformation. When the recuperation of the materials matches the recuperation simulated by the model, this is an indication that the material did not undergo a significant amount of permanent deformation. So, assuming the epoxies can be considered as linear solids, a general Maxwell model as in Fig. 7 applies [11]. This model describes a visco-elastic material that does not have permanent deformation. That is, although there can be a significant amount of creep, the spring in the first branch in Fig. 7 ensures that the material will eventually recuperate to its original shape. The response of such a linear visco-elastic solid to a constant strain is given by:

$\sigma(t)=\left[E_{0}+\sum_{i=1}^{n} E_{i} \cdot \exp \left(\frac{t}{\tau_{i}}\right)\right] \cdot \varepsilon$
Here $E_{0}$ is the 'long term' Young's modulus, $\varepsilon$ is the strain applied and $\tau=\mu / \mathrm{E}$ is the time constant of a spring-dashpot branch. For a more general and accurate model of a visco-elastic solid the number of spring-dashpot branches is infinite and $n$ in Eq. (3) goes to infinity. Thus, further simplification is needed to elaborate the model. In the Alfrey's approximation the response of one branch in the Maxwell model is simplified to a step function instead of an exponential. In this way, a continue relaxation spectrum function can be calculated from the measured data [12].

In another approach, only a finite number of branches is used as the approximation, yielding a Prony series, which is a good assumption for an element in a finite element simulations. This approach is also used in this paper to illustrate that the recorded creep is not all permanent, and that, in first order, we can assume that the material will snap back to retain its original shape.

The parameters of the linear visco-elastic solid with no permanent deformation were determined using the relaxation curve from the creep experiments. The measured stress response was fitted using a Prony series with four exponential terms in order to derive the transfer function of the model:

$$
\begin{aligned}
& H(p)=p \cdot L\left\{\frac{\sigma^{\prime}(t)}{\varepsilon}\right\}=\frac{1}{\varepsilon}\left(A_{0}+\sum_{i=1}^{4} \frac{p A_{i}}{p-T_{i}}\right) \\
& \sigma^{\prime}(t)=A_{0}+\sum_{i=1}^{4} A_{i} \exp \left(T_{i} \cdot t\right)
\end{aligned}
$$

With $H$ the transfer function. $\sigma^{\prime}(t)$ the measured stress in the relaxation experiment, fitted to a four term Prony series, with $A_{i}$ and $T_{i}$ the fit parameters. $L\{\}$ denotes a Laplace transformation and $\varepsilon$ is the applied strain in the relaxation experiment.

Fig. 8a gives the recuperation of the three epoxies. SU-8 and Epocore recuperates more because the remaining stress in these samples are lower then in Epoclad. The tensile test samples do not allow compressive stress, so in order to measure the recuperation some residual tensile stress must be left on the sample. Fig. $8 \mathrm{~b}$ depicts a detailed plot of the simulated versus the measured response of the recuperation. The difference between the simulation and measurement at the minimum, $\Delta d$, can be accounted for by the resolution in the displacement measurement due to a limited sampling rate and gear slack in the actuator.

The behavior of recuperation of the simulated material and the measured recuperation is similar. The simulated recuperation is in fact the recuperation of an analog material that displays, for sure, no permanent deformation. The amount of the recuperation is comparable for the measured and the analog simulated material. This is an indication that the better part of the creep in the epoxies is not a permanent deformation, but that the creep can be recuperated so that the sample will obtains its original shape again. When the material would have no ability to recuperate the undergone creep, the measured curve in Fig. $8 \mathrm{~b}$ would follow the dashed line. The material will still be creeping due to the residual tensile load on the thin film samples. This possibility can also be modeled as a linear solid as in Fig. 7, except that the first branch will be left out in order to have a zero 'long term' Young's modulus as indicated in Eq. (3). The doted line in Fig. $8 \mathrm{~b}$ shows the recuperation when a significant part of the creep was permanent. This can be modeled as a series connection of two Maxwell models, but with one having no first branch and the time constants of the other branches relative high. When these time constants are high enough they would not show in the measurements or only partially. This means that the epoxies can suffer from permanent deformation but it will only occur at very long delays with respect to the applied stress or strain. This is a topic for the further investigation of the reliability of the epoxies for use in MEMS. 

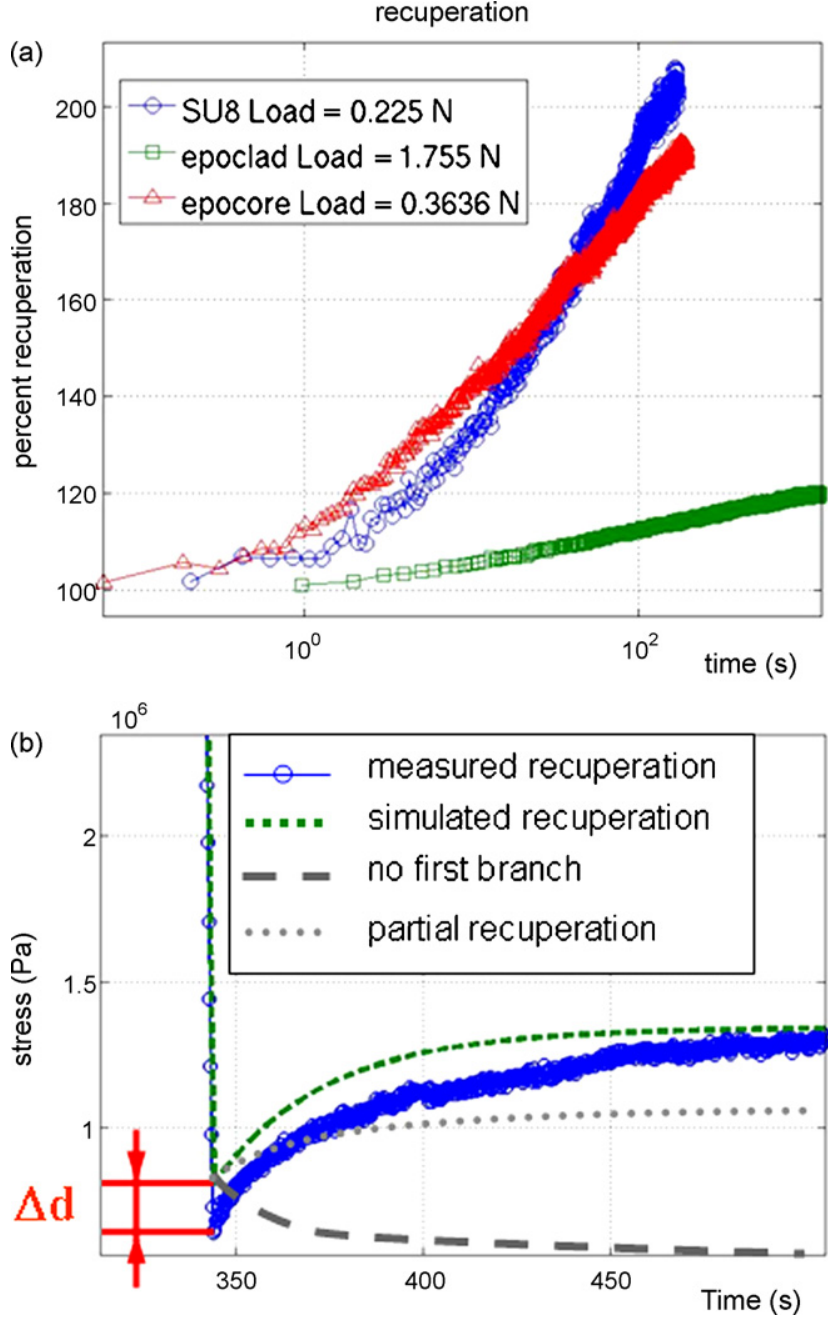

Fig. 8. : (a) Semi-logarithmic plot of the recuperation relative to the remaining tensile stress. (b) Simulated versus measured response of the recuperation behavior of SU-8.

\section{Conclusion}

A direct and fundamental measurement of the in-plane Young's modulus of Epoclad and Epocore is performed, yielding 2.2 and 2.4 GPa for Epoclad and Epocore, respectively. It is demonstrated that the three photo epoxies under investigation show visco-elastic properties by measuring the creep. Although the creep is in first order reversible, this hysteresis effect can significantly influence device performance. It indicates the importance of characterizing the materials' responses at higher frequency domains. Also, the importance of studying the reliability of MEMS using epoxies as structural layer. Because, no clear permanent deformation was recorded in the time interval used, it is possible that it does occur in larger time intervals.

\section{Acknowledgements}

This work has been carried out in the frame of SBO-program project 060046, Gemini, sponsored by the "instituut voor de aanmoediging van Innovatie door Wetenschap en Technologie in Vlaanderen" (IWT), Belgium.

\section{References}

[1] Jonathan M. Engel, Jack Chen, Chang Liu, David Bullen, Polyurethan rubber allpolymer arificial hair cell sensor, J. Microelectromech. Syst. 15 (2006) 729-736.

[2] Yusaku Kato, Tsuyoshi Sekitani, Makoto Takamiya, Masao Doi, Kinji Asaka, Takayasu Sakurai, Takao Someya, Sheet-type Braille displays by integrationg organic field-effect transistors and polymeric actuators, IEEE Trans. Electron Dev. 54 (2007) 202-209.

[3] Po-Jui Chen, D.C. Robger, Rajat Agrawal, Saloomeh Saati, Ellis Meng, Rohit Verma, M.S. Humayun, Yu-Chong Tai, Implantable micromechanical parylenebased pressure sensor for unpowered intraocular pressure sensing, J. Micromach. Microeng. 17 (2007) 1931-1938.

[4] H. Schroder, J. Bauer, F. Ebling, M. Franke, A. Beier, P. Demmer, W. Sullau, J. Kostelnik, R. Modinger, K. Pfeiffer, U. Ostrzinski, E. Griese, Waveguide and packaging technology for optical backplanes and hybrid electrical-optical circuit boards, Orlando (Kissimmee), FL, USA, Proc. SPIE 6124 (2006), 612407-1 April.

[5] F. Ceyssens, M. Driesen, K. Wouters, R. Puers, A low-cost and highly integrated fiber optical pressure sensor system, Sens. Actuators A 145-146 (2008) 81-86.

[6] S.M. Spearing, Material issues in microelectromechanical systems (MEMS), Acta Mater. 48 (2000) 179-196.

[7] E. Arzt, Size effects in materials due to microstructural and dimensional constraints: a comparative review, Acta Mater. 46 (1998) 5611-5626.

[8] Ru Feng, R.J. Farris, Influence of processing conditions on the termal and mechanical properties of SU8 negative photoresist coatings, J. Micormech. Microeng. 13 (2003) 80-88.

[9] J.R. Park, Polymer Science and Technology, second ed., Prentice Hall Books, Upper Saddle River, 2007.

[10] Toshiyuki Tsuchiya, Specimen size Effect on tensile strength of surfacemicormachined polycrystalline silicon thin films, J. Microelectromech. Syst. 7 (1998) 1653-1675.

[11] S.W. Park, R.A. Schapey, Methods of interconversion between linear viscoelastic material functions. Part I - a numerical method based on Prony series, International Journal of Solids and Structures 36 (1999) 1653-1675.

[12] I.M. Ward, J. Sweeney, An introduction to the mechanical properties of solid polymers, John Wiley \& Sons, Ltd, 2004, p. 75.

\section{Biographies}

K. Wouters, was born in Aarschot, Belgium in 1983. He received his Master of engineering degree in 2006. He is now working as a research assistant under prof. Puers. His main research interests is the investigation of the use of polymers as a structural layer in MEMS.

R. Puers recieved his M.S. degree at the Katholieke Universiteit te Leuven, where he obtained his Ph.D. in 1986. The same year, he became Director of the clean room facilities for silicon and hybrid circuit technology at the ESAT-MICAS laboratories at the K.U.Leuven. He was a pioneer in the European research efforts in silicon micromachined sensors, MEMS and packaging techniques, for biomedical implantable systems as well as for industrial devices. In addition, his general interest in low power telemetry systems, with the emphasis on intelligent interface circuits and on inductive power and communication links has promoted the research of the ESATMICAS labortary to international recognition. At present, he is as a full professor at the K.U.Leuven, teaching courses in 'Microsystems and Sensors', 'Biomedical Instrumentation and Stimulation', 'Biomedical Equipment and Regulations', 'Production techniques for microelectronics', and basic courses in 'Electronics, System Control and Information Technology'. He is co-author of more than 450 papers on biotelemetry, sensors, MEMS and packaging in reviewed journals or international conferences. He is a Fellow of the Institute of Physics (UK), senior member of the IEEE, member of the Electon Device Society (EDS) and many others. 